\title{
Modeling of disturbance torque in an aerostatic bearings-based nano-satellite simulator
}

\author{
LIU Yanfang", HUO Mingying, and QI Naiming
}

Department of Aerospace Engineering, Harbin Institute of Technology, Harbin 150001, China

\begin{abstract}
The disturbance torque of aerostatic bearings is in the same order of the reaction wheel, which causes difficulty in evaluation of the designed attitude control strategy of a nano-satellite based on the aerostatic bearing. Two approaches are proposed to model the disturbance torque. Firstly, the gravity induced moment, the vortex torque, and the damping moment are modeled separately. However, the vortex torque and the damping moment are coupled with each other as both of them are caused by the viscosity. In the second approach, the coupling effect is considered. A nano-satellite is constructed based on aerostatic bearing. The time history of the free rotation rate from an initial speed is measured by the gyro, which is further used to calculate the rotation angle and acceleration. The static vortex torque is measured via the removable micro-torque measurement system. Based on these data, the model parameters are identified and modeling errors are presented. Results show that the second model is more precise. The root mean squire error is less than $0.5 \times 10^{-4} \mathrm{~N} \cdot \mathrm{m}$ and the relative error of the static vortex torque is $0.16 \%$.
\end{abstract}

Keywords: aerostatic bearing, disturbance torque, vortex torque, spacecraft simulator, modeling and identification.

DOI: $10.21629 / J S E E .2018 .03 .19$

\section{Introduction}

Due to noncontact mechanism, aerostatic bearings have many important advantages over conventional bearings, such as lower friction, lower noise, lower heat generation, higher speed capability, less contamination, and higher accuracy. As a result, they are extensively studied via experiment and simulation and increasingly applied to precision instrument, inertial instrument, and zero-gravity environment simulation [1-7]. Most of the works focus on calculation methods [8,9], dynamic and static characters

\footnotetext{
Manuscript received April 17, 2017.

*Corresponding author.

This work was supported by the National Natural Science Foundation of China (11672093; 51705109), the Special Foundation of Heilongjiang Postdoctoral Science (LBH-TZ1609), the Open Fund of National Defense Key Discipline Laboratory of Micro-Spacecraft Technology (HIT.KLOF.MST.201507), and the Fundamental Research Funds for the Central Universities (HIT.NSRIF.201622).
}

$[10,11]$, and new aerostatic bearings such as those with small feed holes [12] and vortex bearings [2].

In most applications, the disturbance torque is small enough to be ignored. Thus, seldom efforts are carried out on the modeling and compensation of the disturbance torque. However, the aerostatic bearings are not friction free. The disturbance torque of an aerostatic cylindrical bearing consists of three parts: the gravity induced moment $[13,14]$, the vortex torque [15-18], and the viscosity induced damping moment [19]. If the aerostatic bearing is perfectly manufactured, the air flow is symmetric. Unfortunately, this can be hardly achieved. Machining errors, such as roundness error of the bearing surface, the tilting error of the orifice restrictors and the surface roughness, cause asymmetric air flow in the bearing gap. The non-uniform air flow going through the throttling slit causes tangential distributary and results in a spin torque even when the velocity of the bearing is zero. This spin torque is usually referred to as vortex torque [15-18], which is an important factor of aerostatic bearings. Meanwhile, damping moment is induced by the viscosity of the gas when the aerostatic bearing shaft rotates, which is referred to as viscosity induced damping moment [19]. Actually, the vortex torque and viscosity induced damping moment are always coupled with each other as they are both caused by the viscosity of the air flow. Due to unbalance of mass, the centroid of the rotatable part, including the bearing shaft and the equipment on the shaft, is not on the revolving axis (the axis of the bearing shaft). If the revolving axis is not vertical, the gravity induces a restoring moment, which is referred to as gravity induced moment $[13,14]$.

The disturbance torque cannot be ignored for the hardware-in-loop simulation of the attitude control system of the nano-satellite. A reaction wheel with small output moment is selected as it is light in weight and capable of controling the nano-satellite with small inertia moment. However, the saturated moment of this kind of reaction wheel is also small, in the order of $10^{-3} \mathrm{~N} \cdot \mathrm{m}$, and its smal- 
lest output is less than $10^{-4} \mathrm{~N} \cdot \mathrm{m}$. The disturbance torque of a spacecraft simulator based on an aerostatic bearing is also in the order of $10^{-3}-10^{-4} \mathrm{~N} \cdot \mathrm{m}$ [5]. Thus, to evaluate the effectiveness of the designed attitude control strategy, the disturbance torque should be known precisely to be compensated for exactly.

In this paper, the disturbance torque of the aerostatic bearings is modeled and identified. Two approaches are proposed. In the first approach, the three components are modeled separately. In the second approach, the coupling effect between the vortex torque and the damping moment is taken into consideration. Experiments are carried out to evaluate the effectiveness of the proposed model.

\section{Problem statement}

As shown in Fig. 1, the nano-satellite simulator is supported on a "T"-type air-bearing, which consists of a thrust bearing and a journal bearing. The main components supported on the platform are a power supply system, a reaction wheel, a PC104 type main computer board, a rate gyro, and a wireless communication unit. The saturated moment and angular momentum of the wheel are $10^{-3} \mathrm{~N} \cdot \mathrm{m}$ and $0.08 \mathrm{~N} \cdot \mathrm{ms}$. The minimum output is less than $10^{-4} \mathrm{~N} \cdot \mathrm{m}$. The $1 \sigma$ bias stability of the rate gyro is less than $0.03 \% \mathrm{~h}$, and the nonlinearity of the scalar factor is less than $5 \times 10^{-5}$.

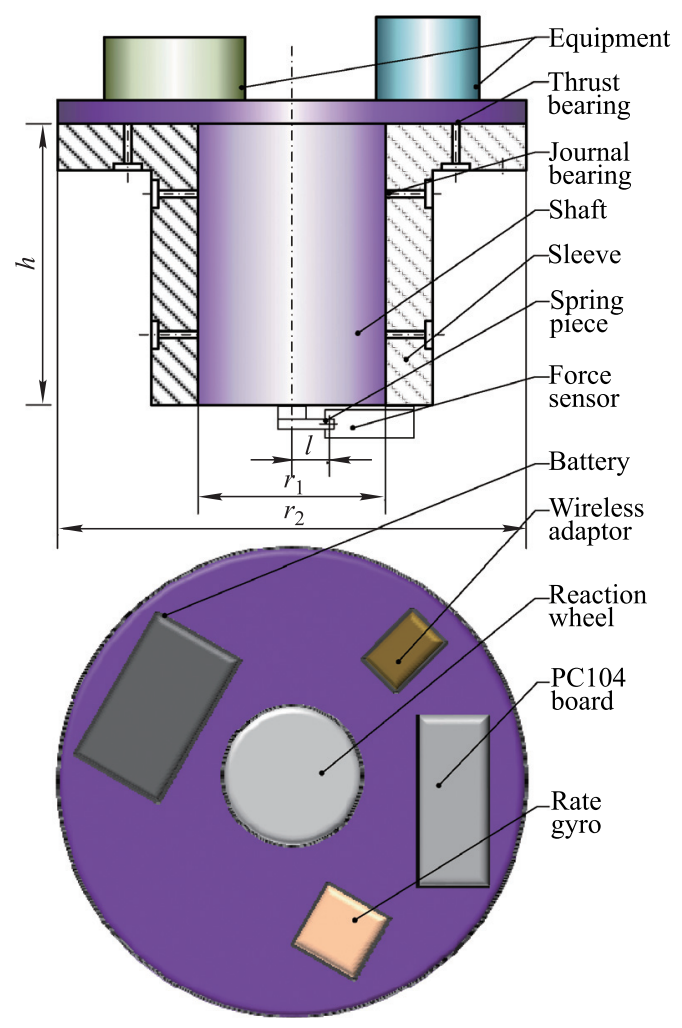

Fig. 1 Nano-satellite simulator based on the "T"-type aerostatic bearing
The simulator is also equipped with a removable microtorque measurement system which consists of a spring piece and a force sensor. The spring piece rotates with the shaft and the sensor is fixed on the sleeve. The torque acted on the shaft balances with the force acted on the sensor.

Usually, the disturbance torque of the air-bearing is in the range of $10^{-3}-10^{-4} \mathrm{~N} \cdot \mathrm{m}$, which is in the same order of the output moment of the reaction wheel. Thus, it can hardly evaluate the designed attitude control system of the nano-satellite via the simulator based on the aerostatic bearing. To overcome this difficulty, the disturbance torque is required to be known precisely and compensated for exactly. In the following sections, the disturbance torque is modeled by two approaches.

\section{Modeling of the disturbance torque}

This section presents two approaches to model the disturbance torque.

\subsection{Approach 1}

(i) Gravity induced moment

As shown in Fig. 2, $y$-axis is the revolving axis. Denote the centroid of the shaft and the equipment on it as $C$. The reference plane is constructed through the point $C$ and perpendicular to $y$-axis and denoted as R-plane. The R-plane intersects $y$-axis at the point $O$. The base plane is the horizontal plane though the point $O$ and denoted as B-plane. The angle from $b$-axis to R-plane is the horizontal angle and denoted as $\gamma$. The gravity induced moment can be expressed [13] as

$$
\begin{gathered}
M_{\mathrm{g}}=-m g r \sin \gamma \sin \psi \\
\psi=\phi+\theta
\end{gathered}
$$

where $m, r$ are the mass and eccentricity, $g$ is acceleration of gravity, $\theta$ is the rotation angle of the bearing, and $\phi$ represents the direction of the eccentricity.

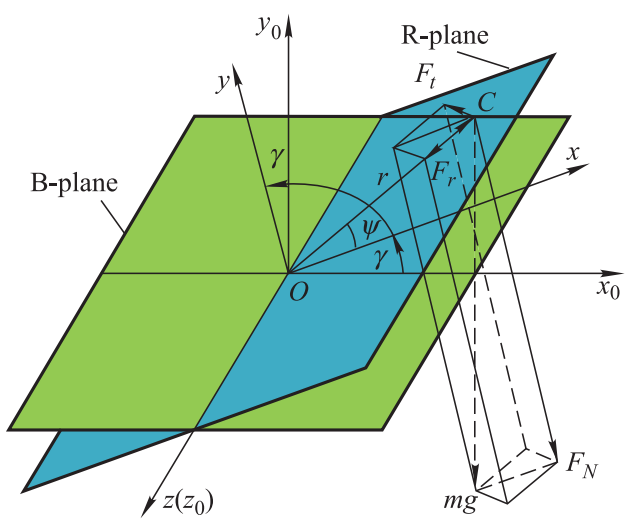

Fig. 2 Illustration of the gravity induced moment 
(ii) Viscosity induced damping moment

The shear stress caused by the viscosity of air can be approached by

$$
\sigma=-\mu \frac{\mathrm{d} V}{\mathrm{~d} n}
$$

where $\mu$ is the coefficient of the viscosity and $\frac{\mathrm{d} V}{\mathrm{~d} n}$ represents the velocity gradient. As shown in Fig. 1, the "T"type aerostatic bearing consists of a thrust aerostatic bearing and a journal aerostatic bearing. Then, the viscosity induced damping moment can be calculated [19] as

$$
M_{\mathrm{d}}=-\frac{\pi \mu \dot{\theta} r_{1}^{4}}{2 n}\left[3\left(\frac{r_{2}}{r_{1}}\right)^{4}+4 \frac{h}{r_{1}}-3\right]
$$

where $n$ is the thickness of the air gap between the shaft and the sleeve.

(iii) Vortex torque

As stated in Section 1, the vortex torque is mainly caused by the machining errors. The relationship between the vortex torque and the machining errors is quite complicated. It is hard to find an analytic expression to model the vortex torque. Fortunately, the machining errors are fixed once the bearing is machined. Thus, the vortex torque is assumed to be constant and denoted by $M_{v}$.

The total disturbance torque is given by the summation of the above three moments

$$
\begin{gathered}
M_{1}=-m g r \sin \gamma \sin (\phi+\theta)- \\
\frac{\pi \mu \dot{\theta} r_{1}^{4}}{2 n}\left[3\left(\frac{r_{2}}{r_{1}}\right)^{4}+4 \frac{h}{r_{1}}-3\right]+M_{v} .
\end{gathered}
$$

\subsection{Approach 2}

As stated above, the vortex torque and the viscosity induced damping moment are coupled with each other. The air flow in the gap between the shaft and the sleeve is quite complicated. However, the asymmetric air flow caused by machining errors and the rotation of the shaft can be equivalent as tangential distributary shown in Fig. 3 .

As shown in Fig. 3(a), if the shaft does not rotate, the tangential distributary is caused by machining error only and distributed almost symmetrically about the center line of the gap. In this case, the torque caused by the tangential air flow is a pure vortex torque and referred to as static vortex torque. If the shaft rotates in the same direction of the effective tangential flow, the speed of the tangential flow increases due to the viscosity. As shown in Fig. 3(b) Fig. 3(d), the speed of the air flow in the side of the shaft increases more rapidly with the rotation rate. In this case, the torque acted on the shaft is caused by both the vortex and the damping moment. The torque is assumed to be a function of the maximum speed of the effective tangential flow, which is affected both by the intensity of the vortex and the rotation of the shaft.

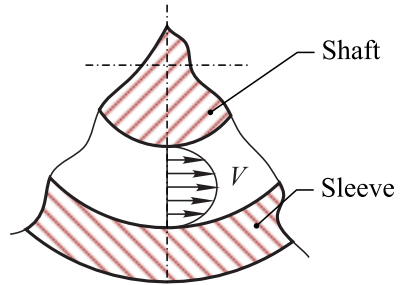

(a) Without rotation

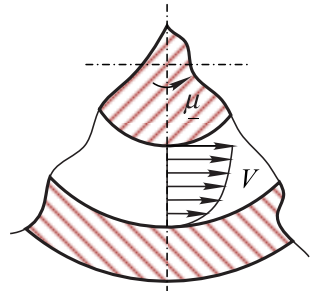

(c) Rotation at a high speed

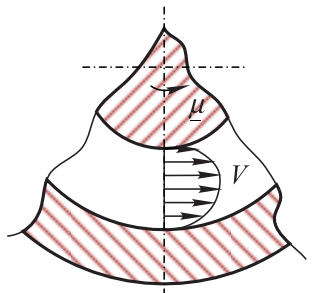

(b) Rotation at a low speed

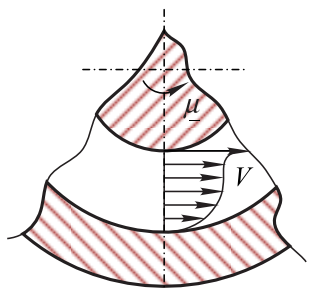

(d) Rotation at a very high speed
Fig. 3 Illustration of the coupling effects between the viscosity induced damping moment and the vortex torque

The maximum speed is assumed to have a linear relation with respect to the rotation rate

$$
v=v_{0}+k_{1} \dot{\theta}
$$

Thus, the torque is approximated by

$$
M_{v d}=a_{1}|v|^{\alpha+k_{2} \dot{\theta}} \operatorname{sign}(v) .
$$

This expression is obtained experimentally. As demonstrated in the next section, this model is effective to describe the coupling effects of the vortex torque and the viscosity induced damping moment. From (7), one can note that the disturbance torque caused by relative movement is not exactly proportional with the relative speed. In other words, it does not obey Newtonian fluid. The total disturbance torque is

$$
\begin{gathered}
M_{2}=-m g r \sin \gamma \sin (\phi+\theta)+ \\
a_{1}\left|v_{0}+k_{1} \dot{\theta}\right|^{\alpha+k_{2} \dot{\theta}} \operatorname{sign}\left(v_{0}+k_{1} \dot{\theta}\right) .
\end{gathered}
$$

\section{Experiment investigation}

\subsection{Data acquisition and preprocessing}

The nano-satellite simulator is constructed based on the configuration given in Section 2. Excite the simulator with an initial rotation speed and leave it to rotate freely (without any external force except the disturbance torque). The rotation speed is measured by the gyro. The angular position and acceleration are calculated via numerical integration and tracking filter [20]:

$$
\left\{\begin{array}{l}
\widehat{\theta}_{k+1}=\widehat{\theta}_{k}+h \widehat{\dot{\theta}}_{k} \\
\widehat{\dot{\theta}}_{k+1}=\widehat{\dot{\theta}}_{k}+h \widehat{\ddot{\theta}}_{k} \\
\widehat{\ddot{\theta}}_{k+1}=\widehat{\ddot{\theta}}_{k}+h \mathrm{fh}\left(\widehat{\dot{\theta}}_{k+1}-\dot{\theta}_{k+1}, \widehat{\ddot{\theta}}, s, h_{0}\right)
\end{array}\right.
$$


where $h$ is the sampling step, $s$ and $h_{0}$ are the designed speed and filter factor, respectively, $\widehat{o}$ represents the estimated value of $o$, and the function $\mathrm{fh}\left(x_{1}, x_{2}, r, h_{0}\right)$ is defined as

$$
\left\{\begin{array}{l}
d=r h_{0} \\
d_{0}=h_{0} d \\
y=x_{1}+h_{0} x_{2} \\
a_{0}=\sqrt{d^{2}+8 r|y|} \\
a=\left\{\begin{array}{l}
x_{2}+\frac{a_{0}-d}{2} \operatorname{sign}(y), \quad|y|>d_{0} . \\
x_{2}+\frac{y}{h_{0}}, \quad|y| \leqslant d_{0}
\end{array}\right. \\
\text { fh }=-\left\{\begin{array}{l}
r \operatorname{sign}(a), \quad|a|>d \\
r \frac{a}{d},|a| \leqslant d
\end{array}\right.
\end{array}\right.
$$

The measured and estimated data are shown in Fig. 4. The estimated angular velocity follows the measured one closely, which demonstrates the effectiveness of the tracking filter.

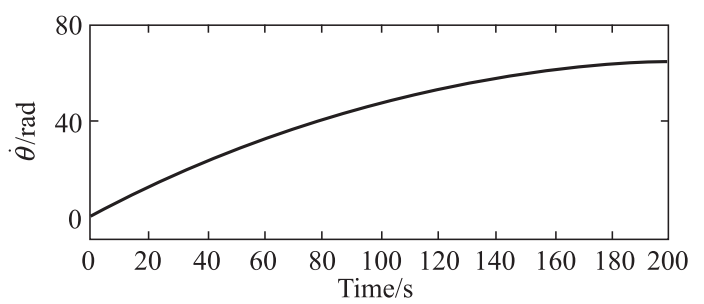

(a) $\dot{\theta}$

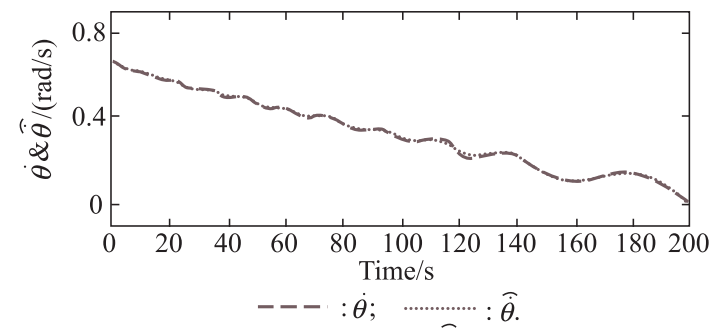

(b) $\dot{\theta} \& \hat{\theta}$

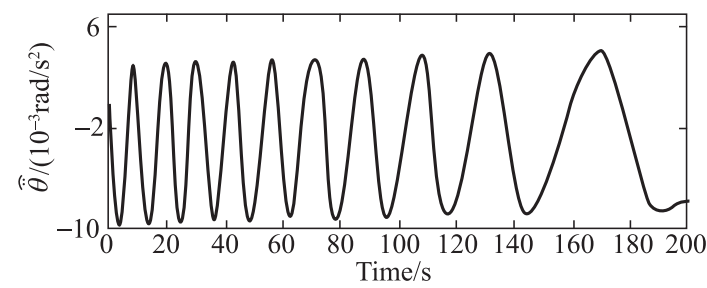

(c) $\ddot{\theta}$

Fig. 4 Illustration of the coupling effects between the viscosity induced damping moment and the vortex torque

The angular speed decreases generally with varyingperiod fluctuates. These fluctuates in the angular speed are mainly caused by the gravity induced moment. The decrease trend is due to that the vortex torque and the damping moment act as resistance. The varying-period fluctuates are obvious in the estimated angular acceleration. One can also observe that the mean value of the angular acceleration slightly decreases in magnitude. The reason is that the damping moment decreases with the angular speed.

The estimated disturbance torque is calculated by

$$
\widehat{M}=J \widehat{\ddot{\theta}}
$$

where $J=0.0896 \mathrm{~kg} \cdot \mathrm{m}^{2}$ is the moment of inertia of the simulator.

\subsection{Evaluation of Approach 1}

Seen from (4), the coefficient of the viscosity induced damping moment is determined by the structural parameters and is calculated as $c=-8.14 \times 10^{-5} \mathrm{~kg} \cdot \mathrm{m}^{2} / \mathrm{s}$. Then the disturbance torque is expressed as

$$
M_{1}=a \sin (\phi+\theta)+c \dot{\theta}+M_{v}
$$

where

$$
\begin{gathered}
a=-m g r \sin \gamma \\
c=-\frac{\pi \mu r_{1}^{4}}{2 n}\left[3\left(\frac{r_{2}}{r_{1}}\right)^{4}+4 \frac{h}{r_{1}}-3\right] .
\end{gathered}
$$

The parameters to be identified are $a, \phi$, and $M_{v}$. They are optimized by solving

$$
\min _{a, \phi, M_{v}}\left\|\widehat{M}-M_{1}\right\|
$$

There are plenty of algorithms that can be used to solve the above problem. In this paper the Matlab function fmincon is employed to search for the optimal parameters.

The identified parameters are $a=-5.48 \times 10^{-4} \mathrm{~N} \cdot \mathrm{m}$, $M_{v, 1}=-2.88 \times 10^{-4} \mathrm{~N} \cdot \mathrm{m}$, and $\phi=-0.775 \mathrm{rad}$. The identified results are shown in Fig. 5. The identified model is also evaluated by using another set of data. The measured angular speed and its estimated value, as well as the estimated angular position and acceleration are shown in Fig. 6 and the results are shown in Fig. 7. The mean value of the estimated disturbance torque increases slightly with time. Noting from that the angular velocity decreases with time, the increase of the mean value is mainly caused by the viscosity induced damping moment. Unfortunately, the model does not capture this feature well. The reason is explained as following. Equation (4) is based on assumption of laminar flow. However, the flow in the gap is much complicated and the gradient of the velocity is not constant. Then the coefficient in (14) is smaller than the real one. To overcome this problem, this damping coefficient is added to the parameter set to be optimized

$$
\min _{a, \phi, c, M_{v}}\left\|\widehat{M}-M_{1 / 1}\right\| .
$$




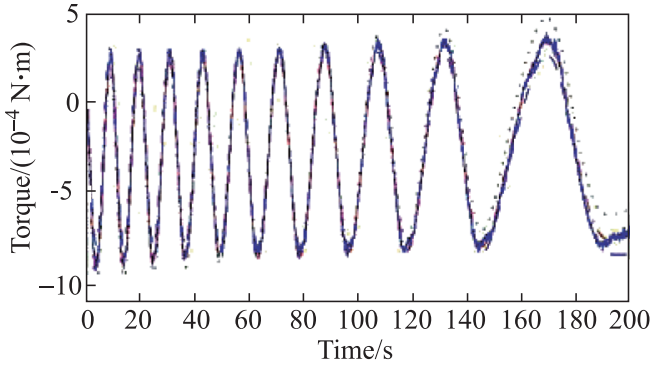

(a) Torque

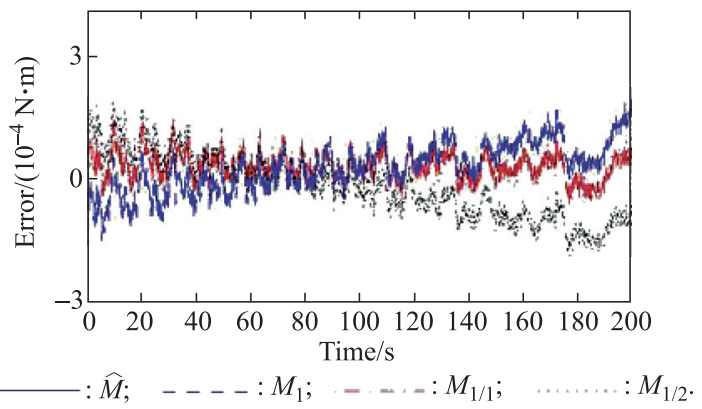

(b) Error

Fig. 5 Identified results of Approach 1

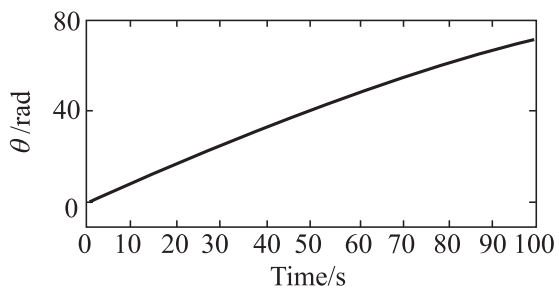

(a) $\theta$

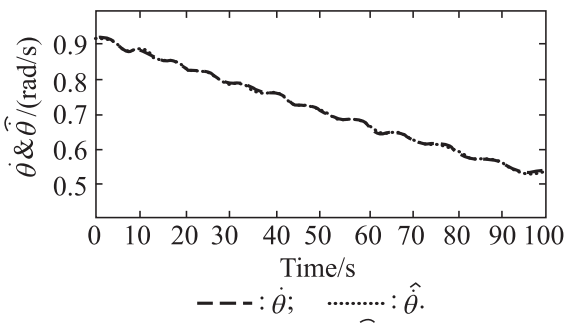

(b) $\dot{\theta} \& \hat{\theta}$

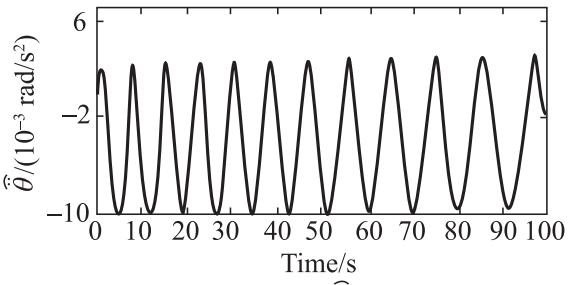

(c) $\widehat{\ddot{\theta}}$

Fig. 6 Data used to evaluate the identified approach 1

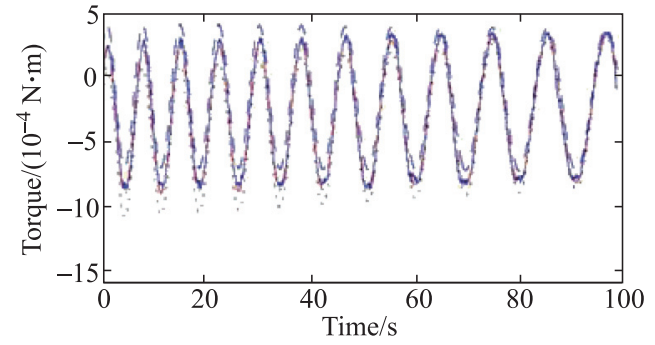

(a) Torque

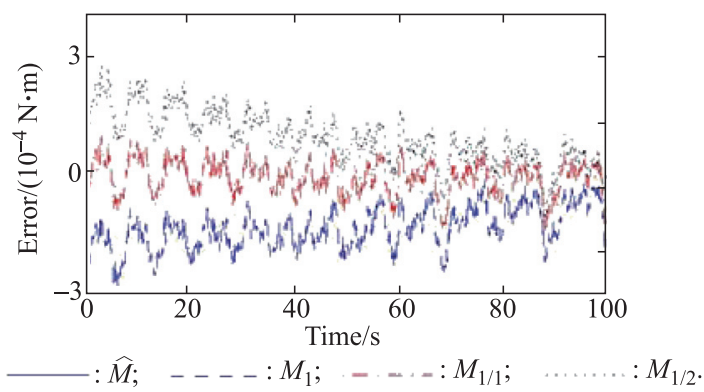

(b) Error

Fig. 7 Performance of the identified approach 1

The identified damping coefficient is $c=-2.49 \times$ $10^{-4} \mathrm{~kg} \cdot \mathrm{m}^{2} / \mathrm{s}$, much greater than the one calculated from (14). Other parameters are identified as $a=-5.59 \times$ $10^{-4} \mathrm{~N} \cdot \mathrm{m}, \phi=-0.786 \mathrm{rad}$, and $M_{v, 1 / 1}=-1.82 \times$ $10^{-4} \mathrm{~N} \cdot \mathrm{m}$. The identified results and evaluation are also shown in Figs. 5-7. The performance is improved significantly.

Actually, the static vortex torque can be measured via the removable micro-torque measurement system. The measured value is $M_{v m}=9 \times 10^{-6} \mathrm{~N} \cdot \mathrm{m}$, which is much smaller than the identified one. To satisfy the constrain of the vortex torque, the object function is modified as

$$
\min _{a, \phi, c, M_{v}}\left\|\widehat{M}-M_{1 / 2}\right\|+\lambda\left|M_{v}-M_{v m}\right|
$$

where $\lambda$ is the weight coefficient. Let $\lambda=100$, the identified parameters are $\phi=-0.796 \mathrm{rad}, a=-5.74 \times$ $10^{-4} \mathrm{~N} \cdot \mathrm{m}, M_{v, 1 / 2}=-8.99 \times 10^{-6} \mathrm{~N} \cdot \mathrm{m}$, and $c=$ $-6.66 \times 10^{-4} \mathrm{~kg} \cdot \mathrm{m}^{2} / \mathrm{s}$. The results are also shown in Figs. 5 - 7. The performance becomes worse due to the vortex torque constrain. Meanwhile, one can observe that the identified gravity induced moment is similar. However, the vortex torque and the damping moment are significantly different. This result implies that this model can hardly capture simultaneously the static vortex torque and the total disturbance torque in the case that the simulator rotates. The reason is that the coupling effect between the vortex torque and the damping moment is ignored.

\subsection{Evaluation of Approach 2}

Similarly, Approach 2 can be written in a standard form

$$
M_{2}=a \sin (\phi+\theta)+a_{1}\left|v_{0}+k_{1} \dot{\theta}\right|^{\alpha+k_{2} \dot{\theta}} .
$$




$$
\operatorname{sgin}\left(v_{0}+k_{1} \dot{\theta}\right) .
$$

The parameters to be identified are $a, \phi, a_{1}, v_{0}, k_{1}, \alpha$, and $k_{2}$. They are optimized by solving

$$
\min _{a, \phi, a_{1}, v_{0}, k_{1}, \alpha, k_{2}}\left\|\widehat{M}-M_{2}\right\|+\lambda\left|M_{2}\right|_{a=0, \dot{\theta}=0}-M_{v m} \mid
$$

where the second term in the right side is the constrain of the static vortex torque. Let $\lambda=100$. The identified parameters are $a=-5.72 \times 10^{-4} \mathrm{~N} \cdot \mathrm{m}, a_{1}=$ $-5.02 \times 10^{-4} \mathrm{~N} \cdot \mathrm{m}, \phi=-0.785, v_{0}=-5.02 \times 10^{-8}$, $\alpha=0.220, k_{1}=0.639 \mathrm{~s}$, and $k_{2}=-0.147 \mathrm{~s}$.

The identified results and evaluation are given in Fig. 8 and Fig. 9. Generally, the errors are much smaller than that of Approach 1. Approach 2 also can capture the static vortex torque and the total disturbance torque simultaneously. The root mean square error (RMSE) is defined as

$$
e_{\mathrm{rms}, i}=\sqrt{\frac{\sum\left(\widehat{M}-M_{i}\right)^{2}}{N}}, \quad i \in\{1,1 / 1,1 / 2,2\}
$$

where $N$ is the length of the data. The relative error of the static vortex torque is defined by

$$
e_{v, i}=\frac{\left|M_{v, i}-M_{v m}\right|}{M_{v m}}, \quad i \in\{1,1 / 1,1 / 2,2\}
$$

where

$$
M_{v, 2}=\left.M_{2}\right|_{a=0, \dot{\theta}=0}
$$

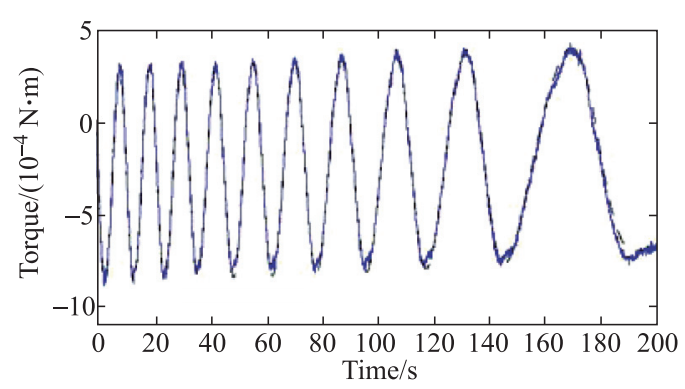

(a) Torque

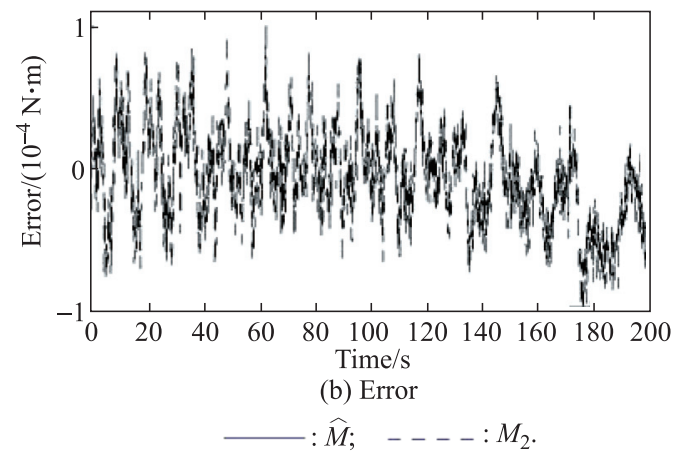

Fig. 8 Identified results of Approach 2

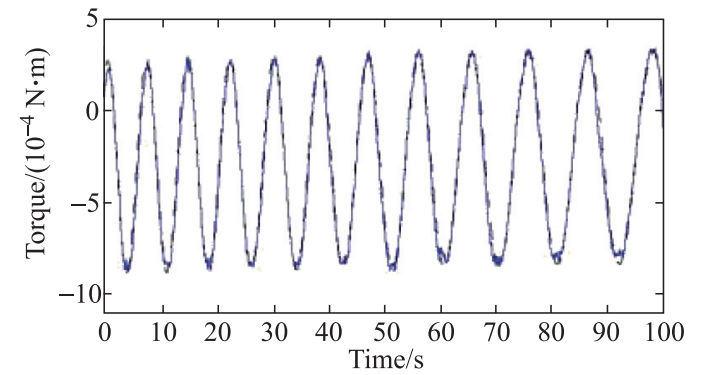

(a) Torque

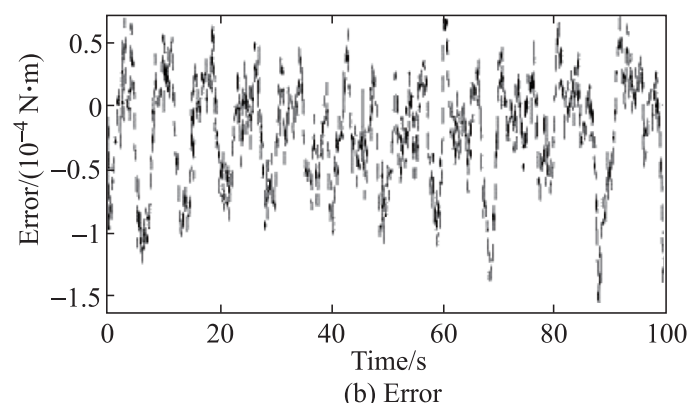

(b) Error

$\longrightarrow: \widehat{M} ; \quad----: M_{1}$.

Fig. 9 Evaluation of the identified Approach 2

These results are summarized in Table 1.

Table 1 Errors of both approaches

\begin{tabular}{ccccc}
\hline Error & $M_{1}$ & $M_{1 / 1}$ & $M_{1 / 2}$ & $M_{2}$ \\
\hline RMSE for data set $1 /\left(10^{-4} \mathrm{~N} \cdot \mathrm{m}\right)$ & 0.720 & 0.429 & 0.943 & 0.382 \\
RMSE for data set $2 /\left(10^{-4} \mathrm{~N} \cdot \mathrm{m}\right)$ & 1.418 & 0.427 & 1.200 & 0.479 \\
Relative error of static vortex torque/\% & 3019 & 1922 & 0 & 0.16 \\
\hline
\end{tabular}

\subsection{Discussion}

The experiment results indicate that the disturbance torque of the aerostatic bearing is mainly caused by gravity induced moment and the viscosity of the gas. Due to machining errors, there exists tangential distributary which results in a spin torque even the angular velocity is zero. This disturbance torque is usually called as vortex torque. However, if the shaft rotates, the vortex torque is coupled with the damping moment induced by the viscosity. Considering the coupling effect, Approach 2 gives a more precise result. The proposed model can be used to calculate the disturbance torque and compensated for it in the control system. This approach can be carried out in the following steps: firstly, the model parameters are identified offline; secondly, the angular position and speed are measured or calculated online; and thirdly, the disturbance torque is calculated and compensated for in the forward loop of the nano-satellite's attitude control system. On the other hand, identified results can also provide a direction on the adjustment of the levelness and eccentricity.

\section{Conclusions}

Two approaches are proposed to model the disturbance torque in the " $\mathrm{T}$ " type aerostatic spring. The first approach 
modeles the gravity induced moment, the vortex torque, and the damping moment separately. Due to the coupling effect of the vortex torque and the damping moment, this approach can hardly capture simultaneously the static vortex torque and dynamic damping moment. Considering the coupling effect, the second approach models the vortex torque and the damping moment as one term. Experiment results show the effectiveness of this approach. The RMSE is less than $0.5 \times 10^{-4} \mathrm{~N} \cdot \mathrm{m}$ and the relative error of the static vortex torque is $0.16 \%$.

\section{References}

[1] LEE K M, DAN E E, TIAN H. Design and control of a spherical air-bearing system for multi-D.O.F. ball-joint-like actuators. Mechatronics, 2002, 13(2): 175-194.

[2] LI X, HORIE M, KAGAWA T. Study on the basic characteristics of a vortex bearing element. International Journal of Advanced Manufacturing Technology, 2012, 64(1-4): 1-12.

[3] CUI P L, ZHANG H J, YAN N, et al. Performance testing of a magnetically suspended double gimbal control moment gyro based on the single axis air bearing table. Sensors, 2012, 12(7): 9129-9145.

[4] INUMOH L O, FORSHAW J L, HORRI N M. Tilted wheel satellite attitude control with air-bearing table experimental results. Acta Astronautica, 2015, 117: 414-429.

[5] SCHWARTZ J L, PECK M A, HALL C D. Historical review of air-bearing spacecraft simulators. Journal of Guidance Control and Dynamics, 2003, 26(4): 513-522.

[6] CHESI S, GONG Q, PELLEGRINI V, et al. Automatic mass balancing of a spacecraft three-axis simulator: analysis and experimentation. Journal of Guidance Control and Dynamics, 2014, 37(1): $197-206$.

[7] XU Z, QI N, SUN Q, et al. Automatic mass balancing of threeaxis rotational spacecraft simulator. Systems Engineering and Electronics, 2015, 37(5): 1124-1132. (in Chinese)

[8] LI Y, DING H. A simplified calculation method on the performance analysis of aerostatic thrust bearing with multiple pocketed orifice-type restrictors. Tribology International, 2012, 56(56): 66-71.

[9] LO C Y, WANG C C, LEE Y H. Performance analysis of high-speed spindle aerostatic bearings. Tribology International, 2005, 38(1): 5-14.

[10] OTSU Y, SOMAYA K, YOSHIMOTO S. High-speed stability of a rigid rotor supported by aerostatic journal bearings with compound restrictors. Tribology International, 2011, 44(1): $9-17$.

[11] NEVES M T, SCHWARZ V A, MENON G J. Discharge coefficient influence on the performance of aerostatic journal bearings. Tribology International, 2010, 43(4): 746-751.

[12] MIYATAKE M, YOSHIMOTO S. Numerical investigation of static and dynamic characteristics of aerostatic thrust bearings with small feed holes. Tribology International, 2010, 43(8): $1353-1359$.

[13] LIN Z, DONG Y. Balance of single axis air bearing table based on its swing characteristics. Review Science \& Technology, 2010, 28(2): 46-49. (in Chinese)

[14] WANG Z, LI Y, BAO G. Study on the mechanics property of three degrees of freedom air-bearing testbed-compensation for the unbalance torque. Chinese Journal of Mechanical En- gineering, 2006, 42(4): 179-184. (in Chinese)

[15] LIU J, SUN Y, LU L, et al, Manufacturing errors effects on disturbing torque of aerostatic bearings. Applied Mechanics and Materials, 2009, 16: 505-509.

[16] LIANG Y, LIU J, SUN Y, et al. Surface roughness effects on vortex torque of air supported gyroscope. Chinese Journal of Aeronautics, 2011, 24(1): 90-95.

[17] YAO Y, DU J, LIU D, et al. Numerical analysis of manufacturing error influence on vortex torque of externally pressurized gas bearings. Acta Aeronautica et Astronautica Sinica, 2003, 24(2): 124 - 128. (in Chinese)

[18] DU J, YAO Y, GAO D, et al. A numericalanalysis of vortex torque of externally pressurized gas journal-thrust bearings. Lubrication Engineering, 2005 (3): 57-59. (in Chinese)

[19] ZHANG Y, SUN N, CAO G. The regularity analysis of low velocity free rotation of single axis air bearing table. Journal of Engineering Design, 2009, 16(6): 436 - 439. (in Chinese)

[20] GAO Z, HUAN Y, HAN J. An alternative paradigm for control system design. Proc. of the 40th IEEE Conference on Decision and Control, 2001: 4578-4585.

\section{Biographies}

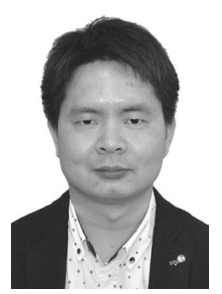

LIU Yanfang was born in 1986. He received his B.S. degree in aircraft design engineering from Harbin Engineering University in 2008 and Ph.D. degree in aeronautical and astronautical science and technology from Harbin Institute of Technology in 2013. He visited and studied in the area of nano-positioning based on piezoelectric actuators in York University in 2013. He is currently an associate professor in Harbin Institute of Technology. His research interests are spacecraft simulator technique and system, dynamics, control and navigation of spacecraft, mechanical and electrical integration and ground simulation of spacecraft, simulation of low gravity environment, smart materials and structures, and shape and vibration control of flexible systems.

E-mail: liu-yanfang@hotmail.com

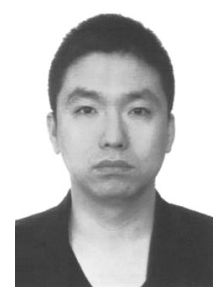

HUO Mingying was born in 1986. He visited and studied in University of Pisa in 2014, and received his Ph.D. degree in aeronautical and astronautical science and technology from Harbin Institute of Technology in 2015 . He is currently a research assistant in Harbin Institute of Technology. His research interests are dynamics and control of electric sail, deep-space exploration, path planning of spacecraft, spacecraft simulator technique and system, and simulation of low gravity environment.

E-mail: huomingying123@163.com

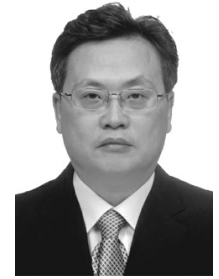

QI Naiming is a professor with School of Astronautics, Harbin Institute of Technology. He received his $\mathrm{Ph}$.D. degree from Harbin Institute of Technology in 2001. His area of research includes aircraft dynamics, guidance, simulation of space environment, and control and integration of electro-mechanical system.

E-mail: qinmok@163.com 